\title{
Correction to: A web-based real-time and full-resolution data visualization for Himawari-8 satellite sensed images
}

\author{
Ken T. Murata ${ }^{1}$ - Praphan Pavarangkoon ${ }^{1} \cdot$ Atsushi Higuchi $^{2} \cdot$ Koichi Toyoshima $^{2}$. \\ Kazunori Yamamoto $^{1}$ - Kazuya Muranaga ${ }^{3}$ - Yoshiaki Nagaya ${ }^{4}$ - Yasushi Izumikawa ${ }^{5}$. \\ Eizen Kimura $^{6} \cdot$ Takamichi Mizuhara $^{7}$
}

Published online: 18 November 2017

(C) Springer-Verlag GmbH Germany 2017

\section{Correction to: Earth Sci Inform}

$$
\text { https://doi.org/10.1007/s12145-017-0316-4 }
$$

The original version of this article, unfortunately, contained errors.

In Tables 3 and 6, there were entries that were not properly aligned on its designated rows.

Table 3 entries "2. 5, 3301 $\times 2701$ and No division" should be aligned with the third row and entries " $2.5,1501 \times 1501$ and No division" should be aligned with the sixth row. In Table 6 entry "Graphic file (PNG)" should be aligned with the fist row and entry "MPEG video file (MP4)" " should be aligned with the sixth row.

The correct Tables are as follows:

The online version of the original article can be found at https://doi.org/ 10.1007/s12145-017-0316-4

Ken T. Murata

ken.murata@nict.go.jp

1 National Institute of Information and Communications Technology, 4-2-1, Nukui-Kitamachi, Koganei, Tokyo 184-8795, Japan

2 Center for Environmental Remote Sensing, Chiba University, Chiba, Japan

3 Systems Engineering Consultants Co., Ltd., Tokyo, Japan

4 International Policy Division, Ministry of Internal Affairs and Communications, Tokyo, Japan

5 Japan Meteorological Agency, Tokyo, Japan

6 Department of Medical Informatics, Ehime University, Ehime, Japan

7 CLEALINKTECHNOLOGY Co., Ltd., Kyoto, Japan 
Table 3 Time interval of Himawari-8 observations and data files provided by JMA: observation area, time interval, data format, data size, and segment

\begin{tabular}{|c|c|c|c|c|c|c|c|}
\hline Area & $\begin{array}{l}\text { Time interval } \\
(\mathrm{min})\end{array}$ & $\begin{array}{l}\text { Spatial resolution } \\
\text { (pixels) }\end{array}$ & Format & $\begin{array}{l}\text { Data size } \\
(\mathrm{MB})\end{array}$ & $\begin{array}{l}\text { Total data size } \\
\text { (GB/day) }\end{array}$ & $\begin{array}{l}\text { Number of files } \\
\text { (files/day) }\end{array}$ & Segments \\
\hline \multirow{2}{*}{ full disk } & \multirow[t]{2}{*}{10} & \multirow[t]{2}{*}{$11,000 \times 11,000$} & $\mathrm{HSF}^{*}$ & 590 & 83 & 22,560 & 10 \\
\hline & & & PNG & 60 & 8.4 & 142 & - \\
\hline \multirow[t]{3}{*}{ Japan Area } & \multirow[t]{3}{*}{2.5} & \multirow[t]{3}{*}{$3301 \times 2701$} & HSF* & 25 & 14 & 9216 & \multirow[t]{3}{*}{ No division } \\
\hline & & & NetCDF & 34 & 19 & 9216 & \\
\hline & & & PNG & 4 & 2.1 & 576 & \\
\hline \multirow[t]{3}{*}{ Target area } & \multirow[t]{3}{*}{2.5} & \multirow[t]{3}{*}{$1501 \times 1501$} & HSF* & 5 & 2.8 & 9216 & \multirow[t]{2}{*}{ No division } \\
\hline & & & NetCDF & 7.8 & 4.4 & 9216 & \\
\hline & & & PNG & 1 & 0.6 & 576 & - \\
\hline
\end{tabular}

*Himawari standard format

Table 6 Data file size and number provided by Himawari-8 real-time web: HR and LR represent high resolution and low resolution, respectively

\begin{tabular}{|c|c|c|c|c|c|c|}
\hline File & Area & Source & $\begin{array}{l}\text { Time interval to } \\
\text { update (min) }\end{array}$ & $\begin{array}{l}\text { Total size/time } \\
\text { step (MB)\# }\end{array}$ & $\begin{array}{l}\text { Size/day } \\
\text { (MB) }\end{array}$ & $\begin{array}{l}\text { Number of files } \\
\text { (files/day) }\end{array}$ \\
\hline \multirow{4}{*}{$\begin{array}{l}\text { Pyramid tile } \\
\text { image file }\end{array}$} & full disk & \multirow[t]{3}{*}{ Graphic file (PNG) } & 10 & 370 & 53,352 & 106,704 \\
\hline & Japan Area & & 2.5 & 27 & 15,840 & 31,680 \\
\hline & Target Area & & - & - & - & - \\
\hline & 16 bands (full disk) & Numerical file (HSF)* & 10 & 59 & 8524 & 426,240 \\
\hline \multirow[t]{3}{*}{ Movie file } & full disk & \multirow[t]{3}{*}{ MPEG video file (MP4) $\dagger$} & 10 & - & $4(\mathrm{HR}) / 2(\mathrm{LR})$ & 2 (with and without coast lines) \\
\hline & Japan Area & & 2.5 & - & $17(\mathrm{HR}) / 8.5$ (LR) & 2 (with and without coast lines) \\
\hline & Target Area & & 10 & - & 7 (HR)/3.5 (LR) & 1 \\
\hline
\end{tabular}

*Himawari standard format

$\dagger$ Moving picture experts group

\# Estimated around the noon (maximum size in a day)

The original article has been corrected. 